\title{
Influence of parameters of a pneumatic grain seeder distributor on the uniform distribution of seeds
}

\author{
Igor I. Ognev ${ }^{1}$, Anton P. Zyryanov ${ }^{2}$, Maksim V. Pyataev,*, and Aleksandr A. Gulyarenko ${ }^{3}$ \\ ${ }^{1}$ Ural Federal University named after First President of Russia B.N. Yeltsin, Ekaterinburg, the \\ Russian Federation \\ ${ }^{2}$ South Ural State Agrarian University, Troitsk, the Russian Federation \\ ${ }^{3}$ Kazakh Agrotechnical University named after S. Seifullin, Astana, R. Kazakhstan
}

\begin{abstract}
The article considers the issue of substantiating the distributing working body parameters for a pneumatic grain seeder. Work on the subject matter is mainly experimental in nature and not sufficiently deeply worked out theoretically. Based on computer simulation, the article determines the most preferred layout of the distributor dividing head, establishes a rational form of the reference cone and the location of the outlet pipes. Based on the simulation of the movement of seed grain particles, the individual design parameters of the distributor dividing head are specified. The results of experimental studies are presented confirming the theoretical background.
\end{abstract}

Today, seeders and sowing machines with pneumatic sowing systems are promising from the point of view of further technical improvement. The advantages of this type of machines, among which there is high productivity, operational and structural manufacturability, were repeatedly expressed in their works by Rakhimov R.S., Astakhov V.S., Kryuchin N.P. [1, 2, 3] and many others. However, a significant drawback of machines with pneumatic sowing systems is high uneven distribution of seed grain over the coverage. In real operating conditions, it can exceed the agrotechnical standard (3\% for seeds) by two, three or more times. In addition to other adverse factors, the transverse unevenness of sowing can significantly reduce the yield of the cultivated crop.

At present, both in Russia and abroad, they have been and are engaged in increasing the transverse uniformity of sowing with pneumatic seeders. To a greater extent, research on the subject under discussion is united by their mainly experimental orientation. Since the process of seed distribution is rather complicated for a theoretical description, in some cases, researchers are limited to experimental verification of the effectiveness of individual technical solutions aimed at increasing sowing uniformity. Thus, the works by A. Yatskul and F. Cointault $[4,5]$ experimentally examined a set of factors affecting the quality of seed distribution by vertical distributors, in particular, factors such as the distributor angle and the supply pipe configuration were identified and experimentally analyzed. Also, the

\footnotetext{
* Corresponding author: $\underline{55 \text { maxim@mail.ru }}$
} 
process of pneumatic transportation of seed in the dispenser is considered by the method of high-speed photography.

The works by Kryuchin N.P. [6], Astakhov V.S. [7], Korobkin I.O. [8] reflected various technical solutions to increase the uniformity of seed grain distribution by horizontal type distributors. Thus, Kryuchin N.P. presented rod diffusers, Astakhov V.S. - teardrop-shaped reflectors, and Karobkin I.O. evaluated plate guides. Mostly the effectiveness of all these technical solutions is verified experimentally in laboratory settings.

Thus, at present, there is no single methodology for the study and justification of the parameters of pneumatic seed drills, which complicates the engineering work on the design of these working bodies. In this regard, the issue has both scientific and practical relevance.

The vertical type seed distributor of a pneumatic seeder consists mainly of two main elements: the supply pipe and the dividing head.

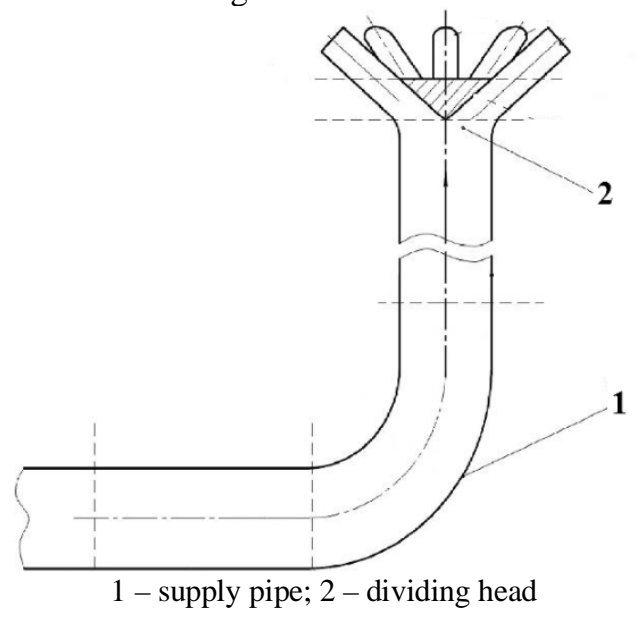

Fig. 1. Seed distributor.

The task of the supply pipeline in the context of increasing seeding uniformity is to ensure the supply of the air mixture (mixture of air and seed grain) to the dividing head with a concentration aligned over the cross section. For this purpose, centering and turbulizing structural elements for the air mixture can be used in its design.

The dividing head parameters as evidenced by works $[4,5,6]$ to an even greater extent affect the distribution uniformity. The lack of theoretical elaboration of the issue led in many respects to a significant number of head designs used on serial sowing machines.

Considering many factors affecting the distribution uniformity, the first thing you need to start a theoretical study with is to decide on the general layout of the dividing head. For this purpose, the process of air flow in the dividing head can be modeled in FlowVision application package. For research purposes, the air flow movement process was modeled in the most common pneumatic seed seeder heads that are already used on serial machines or can presumably be effective (Fig. 2). The heads with and without a reference cone having a rectilinear and curvilinear generators were considered. 

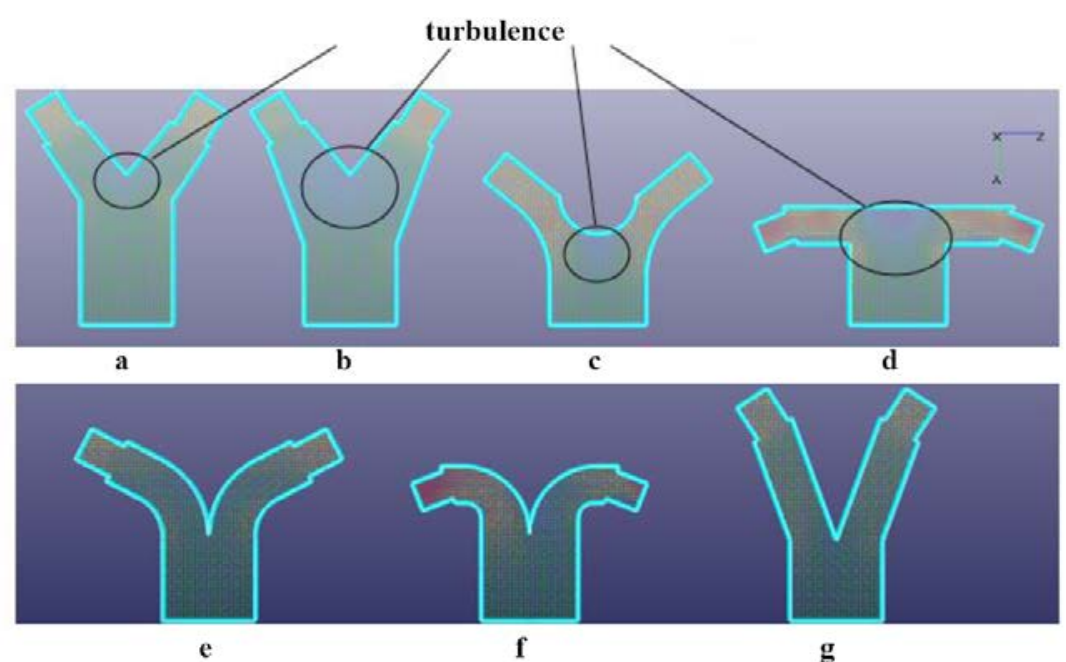

a, b, g - cone with a rectilinear generatrix; c - spherical divider; $d$ - head without the reference cone; e, $\mathrm{f}$ - reference cone with a curved generatrix

Fig. 2. Simulation Results in FlowVision.

The optimality criterion for choosing the configuration of a dividing head was the minimum of its hydraulic resistance expressed in a decrease in the air flow rate, as well as the absence of swirl zones. These criteria have a significant impact on the uniformity of seed grain distribution, as noted in [9]. It is assumed that in the dividing heads that meet the specified criteria, the air mixture will be delayed for a minimum amount of time, thereby it is possible to reduce the influence of other negative factors such as difference in resistance of the grain tube, structural vibrations, etc. on the uniformity of distribution.

Based on the obtained graphical interpretation of the simulation results, we can conclude that the dividing heads with conical reference cones and outlet pipes located to the vertical axis at an angle of at least $120^{\circ}$ (positions e, g in Fig. 2) meet the above criteria. Based on the simulation results, it was found that the air flow velocity in these heads decreases by no more than $25 \%$, while there are no zones of air flow swirl.

Having decided on the preferred layout option for the dividing head - the head with a conical reference cone having a curved generatrix and an angle of installation of the outlet pipes to the vertical axis of $120^{\circ}$. Next, it is necessary to study in more detail the possible effect on the uniformity of distribution of individual structural elements, in particular, the reference cone.

Based on the previous reasoning, we can conclude that for an even distribution of seed grain, the dividing head should have the smallest possible effect on the solid component of the air mixture (seed particles), that is, smoothly redirecting it from the inlet pipe to the outlet pipes, while the particle velocity should be reduced to a minimum. Thus, the inner surfaces of the dividing head and, in particular, the reference cone should provide the minimum possible reduction in the speed of incoming particles of seed grain. This is ensured by the aerodynamic shape of the head and the reference cone parameters.

To determine the rational shape of the reference cone, we will simulate the seed grain movement in the head under the influence of air flow. For this, it is necessary to analyze the nature of the seed grain particle motion at the moment of interaction with the cone, as well as immediately after contact with it before exiting into the outlet pipe. For analysis, the whole process can be divided into three stages:

1) impact interaction of a particle with a reference cone; 
2) particle rebound from the reference cone and movement under the action of the air flow;

3) sliding on the reference cone surface.

\section{1 stage}

Interaction of a seed grain particle with a reference cone has an impact character, in this regard, in accordance with the theorem on the change in the momentum:

$$
m \bar{v}-m \bar{u}=\bar{S},
$$

where $\bar{S}=\int_{0}^{t_{\mathrm{y}}} \bar{P}_{\mathrm{y}} d t$ - shock impulse; $m$ - seed weight; $\bar{v}$ - seed speed after impact; $\bar{u}$ seed speed before impact.

And also taking into account the case of oblique impact (Fig. 3), the particle velocity after interaction with the cone surface can be calculated as:

$$
v=\sqrt{v_{n}^{2}+v_{\tau}^{2}}
$$

where $v_{\mathrm{n}}$ - normal velocity component after impact, $\mathrm{m} / \mathrm{s} ; v_{\tau}$ - tangential component of velocity after impact, $\mathrm{m} / \mathrm{s}$.

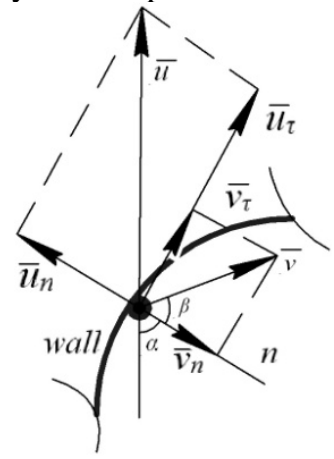

Fig. 3. The impact scheme of the seed grain particles on the curved wall of the reference cone

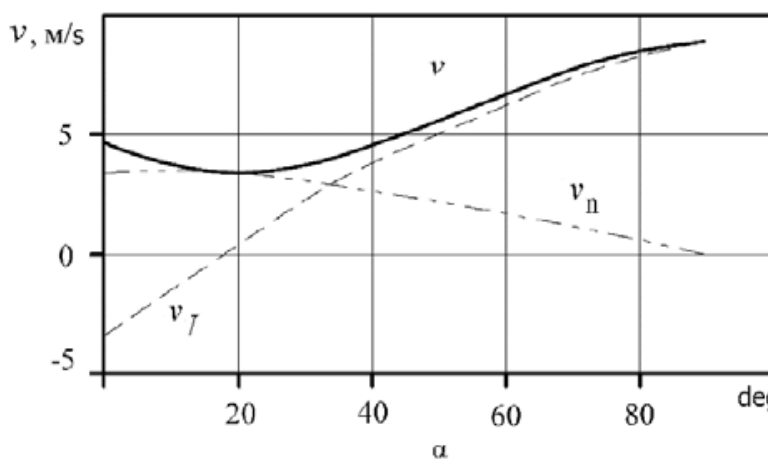

Fig. 4. Change in velocity and its components after impact interaction of a particle with a wall depending on the angle of incidence

The speed components are determined by the following expressions:

$$
\begin{gathered}
v_{n}=k_{n} u \cos \alpha, \\
v_{\tau}=u\left(f \cos \alpha\left(k_{n}-1\right)+\sin \alpha\right),
\end{gathered}
$$

where $\alpha$ - angle of incidence, deg.; $k_{n}$ - recovery factor; $u$ - величина скорости частицы до взаимодействия с конусом, м/c. particle velocity before interaction with the cone, $\mathrm{m} / \mathrm{s}$.

Using dependences (2), (3) and (4), we construct a graphical interpretation of the change in velocity and its components after impact, depending on the angle of incidence $\alpha$ (Fig. 4).

Based on the analysis of Fig. 4, it can be summarized that the speed of the seed grain particle decreases minimally at incidence angles of at least $60^{\circ}$. Thus, the initial conditions to ensure uniform distribution correspond to reference cones that can provide these or large incidence angles.

2 stage 
At the second stage, the seed grain particle is reflected and its further movement in the field of the air flow action and gravity (Fig. 5). The equation of motion of the particle will have the following form:

$$
\left\{\begin{array}{l}
m \frac{d v_{x}}{d t}=m g \cos \beta \\
m \frac{d v_{y}}{d t}=F_{a}-m g \sin \beta
\end{array}\right.
$$

where Fa - air flow force acting on the seed particle, $\mathrm{H}$; $\mathrm{m}$ - mass, kg; $\beta$ - angle of reflection, degrees

After integration (5), expressions are obtained for determining the velocity of the reflected particle and its trajectory:

velocity:

$$
\left\{\begin{array}{l}
v_{x}=v_{\mathrm{B}}-\sqrt{\frac{g \cdot \sin \alpha}{k_{\Pi}}} \operatorname{th}\left(\operatorname{Arth} \sqrt{\frac{k_{\Pi}}{g \sin \alpha}}\left(v_{\mathrm{B}}-v_{0 x}\right)+\sqrt{k_{\Pi} \cdot g \sin \alpha} \cdot t\right), \\
v_{y}=-g t \cos \alpha+v_{0 y}
\end{array}\right.
$$

trajectory:

$$
\left\{\begin{array}{l}
x=v_{\mathrm{B}} t-\frac{1}{k_{\Pi}} \ln \frac{\operatorname{th}\left(\operatorname{Arth} \sqrt{\frac{k_{\Pi}}{g \sin \alpha}}\left(v_{\mathrm{B}}-v_{0 x}\right)+\sqrt{k_{\Pi} \cdot g \sin \alpha} \cdot t\right)}{\operatorname{th}\left(\operatorname{Arth} \sqrt{\frac{k \Pi}{g \sin \alpha}}\left(v_{\mathrm{B}}-v_{0 x}\right)\right)} . \\
y=-\frac{g t^{2}}{2} \cos \alpha+v_{0 y} t
\end{array}\right.
$$

Using expressions (6) and (7), taking the results of previous calculations as initial conditions, we construct the trajectory of the seed grain particle in the transformed coordinates (Fig. 6).

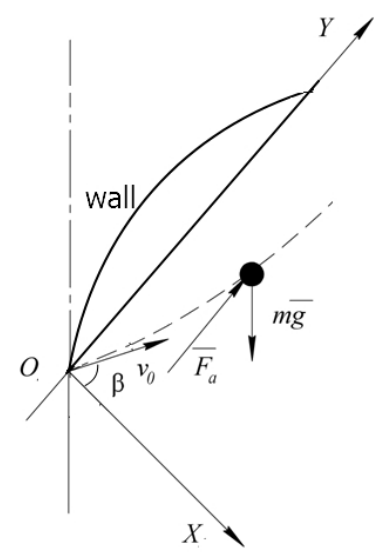

Fig. 5. The scheme of particle motion after interaction with the cone surface

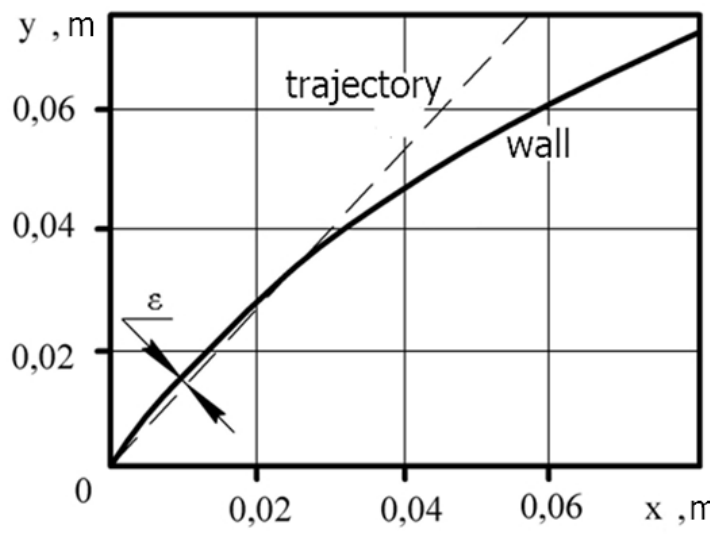

Fig. 6. Particle trajectory after interaction with the cone surface

Based on the analysis of the trajectory, it can be noted that at incidence angles of $60^{\circ}$ or more, an actual rebound of the seed grain particle from the cone surface does not occur (Fig. 6). This is due to the fact that, as a result of the action of the air force, the distance of 
the flow between the trajectory and the surface of the cone $\varepsilon$ does not exceed the linear dimensions of the seed. Thus, at the indicated incidence angles (60 degrees or more), after the interaction of the seed particle with the reference cone, its glide over the surface of the latter is most likely.

\section{3 stage}

Given the nature of the force acting on the seed grain particle (Fig. 7), we will compose the equation of its motion on the surface of the reference cone having a radius of curvature $\mathrm{R}$ :

$$
\left\{\begin{array}{l}
m \frac{d v}{d t}=-m g \sin \alpha-f N \\
m \frac{v^{2}}{R}=N-m g \cos \beta
\end{array}\right.
$$

Having solved the equations (8), we will obtain an expression for determining the particle velocity at any moment of time when it is sliding along a curved surface:

$$
v=\sqrt{\frac{v_{0}^{2}}{\exp \{2 f \varphi\}}+\frac{2 R g}{4 f^{2}+1}\left(\frac{\left(2 f^{2}-1\right)}{\exp \{2 f \varphi\}}-3 f \sin \beta-2 f^{2} \cos \varphi+\cos \varphi\right)},
$$

where $\mathrm{f}$ - coefficient of friction; $\varphi$ - angular coordinate.

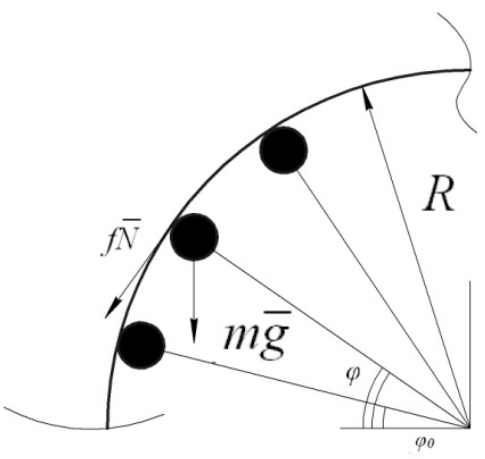

Fig. 7. The scheme of particle motion along the curved surface of the reference cone

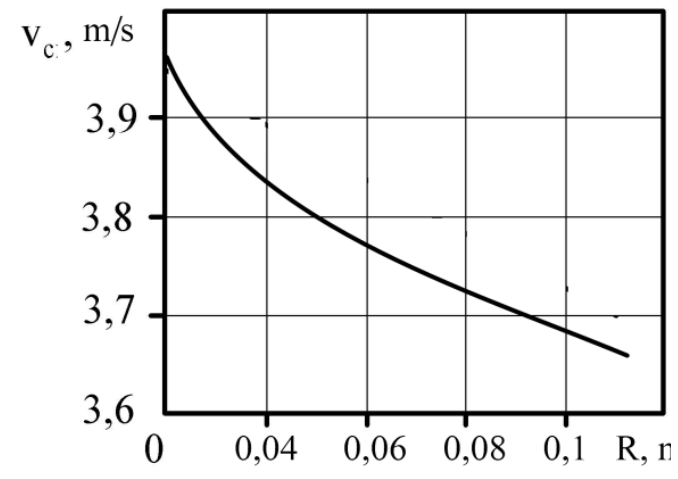

Fig. 8. The nature of the speed change depending on the curvature radius of the generatrix of the reference cone

Thus, based on the results of theoretical studies, it was found that the speed of the particles of seed will decrease minimally with a larger radius of curvature of the generatrix of the dividing cone (Fig. 8).

Based on the simulation results, it can be concluded that for uniform distribution, it is necessary that the dividing head has a reference cone with a curved generatrix of the surface of the largest possible radius and outlet pipes installed with respect to the flow at an angle of at least 120 degrees.

In order to verify the results of theoretical studies, experimental studies were performed on the setup (Fig. 9). 


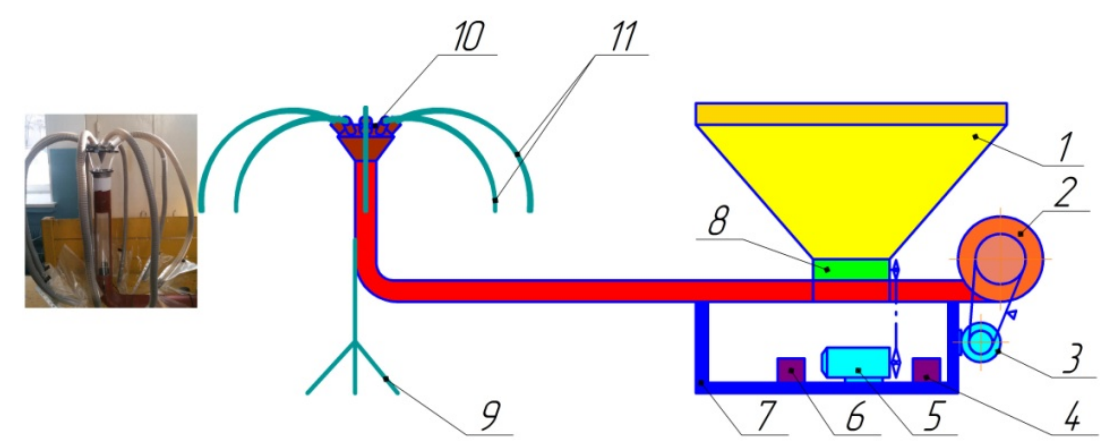

1 - hopper; 2 - fan; 3 - fan motor; 4 - frequency converter of fan frequency adjustment; 5 - electric drive of the metering coil; 6 - frequency converter of adjusting the rotation speed of the metering coil; 7 - installation frame; 8 - metering coil body; 9 - tripod; 10 - dividing head; 11 - grain tube

Fig. 9. Experimental setup.

During the experiments, the technological parameters - air flow rate $\left(v_{\mathrm{B}}, \mathrm{m} / \mathrm{s}\right)$ and persecond seed supply to the system $(\mathrm{q}, \mathrm{kg} / \mathrm{s})$ were taken constant. The air flow rate was set equal to $v_{\mathrm{B}}=25 \mathrm{~m} / \mathrm{s}$ and was controlled using a micromanometer, the per-second seed supply was set to $0.25 \mathrm{~kg} / \mathrm{s}$.

Design parameters - the curvature radius of the reference cone generatrix $(\mathrm{R}, \mathrm{m})$ and the installation angle of the outlet pipes of the head to the pipeline vertical axis $(\gamma$, deg) were varied. The radius of curvature $\mathrm{R}$ during the experiments took the following three values of $0.4 \mathrm{~m}, 0.6 \mathrm{~m}$ and $0.8 \mathrm{~m}$. The installation angle of the pipes $\gamma$ took values of 90 , 105 and 120 degrees.

The variation coefficient $k v$ was used as an optimization criterion, reflecting the uneven distribution of seed grain.

Based on the number of controlled factors, nine experiments were carried out, in triplicate. Each experiment included preparatory work - adjustment of technological parameters, installation of design parameters and direct sowing of a given sample of wheat seeds (2500 grams). The sown seeds were collected in sumps, weighed, after that the variation coefficient was determined.

All identified factors have a significant impact on the optimization criterion. Based on the results of the experiments, the following graph was constructed (Fig. 10).

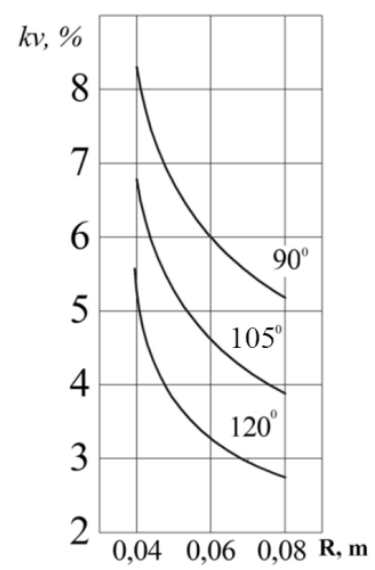

Fig. 10. Uniform distribution of seed grain depending on the design parameters of the dividing head. 
The best uniformity of distribution can be achieved by using a reference cone having a radius of curvature of $0.08 \mathrm{~m}$, that is, the largest in the experiment. In this case, the uneven distribution is $2.68 \%$. A positive effect on uniformity is also exerted by an increase in the installation angle of the outlet pipes of the dividing head.

Conclusions

1. Based on the simulation, it is found that to ensure an even distribution of seed grain, the dividing head of the distributor should have a rational aerodynamic shape that ensures minimal turbulization of the incoming air mixture.

2. It is analytically established that the best prerequisites for uniform distribution are ensured by using a reference cone having a curvilinear forming surface with a radius of curvature and outlet pipes deployed at an angle to the vertical.

3. The adequacy of theoretical studies has been experimentally confirmed. The smallest non-uniformity of sowing of $2.68 \%$ is achieved when using a conical reflector with a generatrix radius of $0.08 \mathrm{~m}$ and an installation angle of outlet pipes $120^{\circ}$.

\section{References}

1. Mudarisov S.G., Gabitov I.I., Rakhimov R.S., Lobachevskiy Y.A., Mazitov N.K., Rakhimov Z.S., Rakhimov I.R., Galimov A.L., Yamaletdinov M.M., Mukhametdinov A.M. Reasoning of modular-type tillage and seeding machines construction diagram and parameters // Journal of the Balkan Tribological Association. 2019. V. 25. No. 3. pp. 695-707.

2. Nemtinov V., Kryuchin N., Kryuchin A., Nemtinova Y. Design and study of seeding devices for small selection seeding machines / /In the collection: E3S Web of Conferences 2019.

3. Astakhov V.S., Petrovets V.R. Block-modular method for constructing wide-sowing sowing units // Vestnik Belorusskoj gosudarstvennoj sel'skohozjajstvennoj akademii. [Bulletin of the Belarusian State Agricultural Academy]. 2019. No 2. pp. 272-274.

4. Yatskul A., Lemière J.P., Cointault F. Influence of the divider head functioning conditions and geometry on the seed's distribution and accuracy of the air-seeder // Biosystems Engineering. - 2017. - No. 161. - pp. 120-134.

5. Yatskul A.I., Lemière J.P. Experimental determination of flow concentration for pneumatic conveying systems of air-seeders // INMATEH-Agricultural Engineering. 2014. - No. 44(3). - pp. 17-24.

6. Astakhov V.S. Analysis of pneumatic centralized sowing systems // Tractors and agricultural machines. - 1997. - No. 10. - pp. 33-34.

7. Kryuchin N.P. Development of a pneumatic seeder for sowing herbs-phytomeliorants // In the collection: Tehnogennaja i prirodnaja bezopasnost' materialy IV Vserossijskoj nauchno-prakticheskoj konferencii [Technogenic and natural safety materials of the IV All-Russian Scientific and Practical Conference]. Saratov State Agrarian University named after N.I. Vavilova Publ. 2017. pp. 253-257.

8. Shevchenko A.P., Korobkin I.O. Effect of the angle of the seeder on the uniform distribution of seeds among the openers // Vestnik Omskogo gosudarstvennogo agrarnogo universiteta [Bulletin of the Omsk State Agrarian University]. 2015. No. 1 (17). pp. 62-65.

9. Pyatayev M.V., Zyryanov A.P. Determination of the rational parameters of the dividing head of a pneumatic grain seeder // Vestnik KrasGAU [Bulletin of KrasSAU]. 2018. No. 3 (138). pp. 88-94. 